\title{
Sarcopenia is Associated with Non-motor symptoms in Han Chinese Patients with Parkinson's Disease: A Cross-Sectional Study
}

\section{Qiu-wan Liu}

Second Affiliated Hospital of Soochow University

\section{Cheng-jie Mao}

Second Affiliated Hospital of Soochow University

Zhao-hui Lu

Second Affiliated Hospital of Soochow University

Rong-fang Shi

Second Affiliated Hospital of Soochow University

\section{Ying-chun Zhang}

Second Affiliated Hospital of Soochow University

\section{Ping Zhao}

Second Affiliated Hospital of Soochow University

Chun-Feng Liu ( $\nabla$ liuchunfeng@suda.edu.cn )

Second Affiliated Hospital of Soochow University https://orcid.org/0000-0002-8364-0219

\section{Research Article}

Keywords: Parkinson's disease, sarcopenia, fatigue, sleep quality, older adults, non-motor symptoms

Posted Date: March 1st, 2022

DOI: https://doi.org/10.21203/rs.3.rs-1314084/v1

License: (c) (i) This work is licensed under a Creative Commons Attribution 4.0 International License. Read Full License 


\section{Abstract}

Background: Sarcopenia is commonly seen in the older adults and increases in incidence with age, also in Parkinson' s Disease (PD). There may be a vicious circle between the development of PD and sarcopenia, but the relationship between the two is not clear. We aimed to investigate the incidence of sarcopenia in patients with PD and the association between non-motor symptoms (NMS) in PD.

Methods: The study included 123 patients with PD and 38 age- and sex-matched healthy controls. All participants were evaluated for sarcopenia using the 2019 Asian Sarcopenia Diagnostic Criteria, and patients with PD underwent standard assessments of motor and non-motor symptoms. Multiple logistic regression and receiver operating characteristic (ROC) curve analyses were used to examine the association between sarcopenia and NMS in PD patients.

Results: The incidence of sarcopenia was significantly higher in patients with PD than in healthy controls ( $26.8 \%$ vs. $10.4 \%, p=0.035$ ). Multiple logistic regression analysis revealed that poorer sleep quality (odds ratio [OR]: 1.305; 95\% confidence interval [Cl]: $1.026-1.622 ; p=0.030)$ and fatigue (OR: $1.097,95 \% \mathrm{Cl}$ : $1.003-1.199, p=0.042$ ) were independently associated with sarcopenia. ROC analysis indicated that the optimal cut-off value for Pittsburgh Sleep Quality Index (PSQI) scores was 10, with 72.7\% sensitivity and $74.4 \%$ specificity (area under the curve [AUC] $=0.776,95 \% \mathrm{Cl}: 0.692-0.846, p<0.001$ ). The optimal cut-off value for Fatigue Severity Scale (FSS) scores was 39 , with $87 \%$ sensitivity and $50 \%$ specificity (AUC=0.725, 95\% Cl: $0.637-0.802, p<0.001$ ). joint use of FSS and PSQI increased the predictive value $(\mathrm{AUC}=0.804, p<0.001)$.

Conclusion: Patients with PD are more susceptible to sarcopenia than healthy older adults. Combined use of FSS and PSQI scores may be a valuable screening method for identifying sarcopenia in patients with PD.

\section{Background}

PD scores the second place of the most common neurodegenerative disease worldwide and is among the conditions exhibiting the fastest increases in morbidity, disability, and mortality[1]. PD is considered to be closely related to aging[2]. According to Chinese epidemiological data, the incidence of PD in individuals over 65 years old is $1.7 \%$. The disease is mainly characterised by static tremor, bradykinesia, muscle rigidity, and postural balance disorders, although it is accompanied by various non-motor symptoms including sleep disorders, cognitive dysfunction, autonomic abnormalities, mood disturbances, and pain. These NMS occur $60-100 \%$ of patients with PD and may play a greater impact on quality of life than motor symptoms[3, 4].

Sarcopenia is an age-related skeletal muscle pathology characterised by reduced muscle mass and muscle strength together with physical performance decline. Although it has something with the aging process, sarcopenia is more commonly observed in older adults with chronic diseases such as Parkinson's syndrome[5-8]. Indeed, previous studies have demonstrated that the prevalence of 
sarcopenia was significantly higher in patients with PD than in the older adults[9]. Sarcopenia has recently been classified as a geriatric syndrome[10] and is closely related to reduced quality of life, impaired cardiopulmonary function[11], falls, and fractures[12]. These in turn lead to increases in the frequency of emergency visits, hospitalization, and mortality in older adults[13].

Studies have indicated that the development of sarcopenia in patients with PD may be related to both motor and NMS[14]. Several groups have reported that sarcopenia is associated with disease duration and stage, as well as Unified Parkinson's Disease Rating Scale (UPDRS)-I/II scores, depression, and cognition[14-17]. However, other studies have reported controversial results[18], and there are large differences in the incidence of sarcopenia in different countries[19, 20]. Furthermore, as far as we know, few studies have investigated sarcopenia in Asian populations, and none have reported sarcopenia data for patients with PD in China.

Therefore, in the present study, we studied the incidence of sarcopenia in patients with PD as well as the association between NMS and PD risk using 2019 Asian Sarcopenia Diagnostic Criteria. Our findings might open a new sight into the importance of sarcopenia in the context of PD, which is essential for improving prognosis in this population.

\section{Methods}

\section{Study participants}

We collected data for consecutive patients treated in the neurology ward and outpatient clinic of the Second Affiliated Hospital of Soochow University from October 2020 to April 2021. The inclusion criteria were as follows: (1) the diagnosis of PD in accordance with the 2016 Chinese Parkinson's Disease Diagnostic Criteria; (2) willingness to undergo assessments with each scale. Patients meeting the following criteria were not included of the study: (1) Parkinson's syndrome or Parkinson's superimposed syndrome stemmed from encephalitis, cerebrovascular disease, poisoning, trauma, drugs, etc.; (2) prior treatment with deep brain electrical stimulation; (3) diagnosis of severe anxiety, depression, schizophrenia, cancer, or physical disease. The control group included healthy older adults who had undergone physical examination at the same centre during the same period. All participants should provide written informed consent in person, and the study won the approval of the Ethics Review Committee of The Second Affiliated Hospital of Soochow University. All procedures were conducted by the ethical standards of the institutional and/or national research committee, following the guidelines of the 1964 Helsinki Declaration as well as its later amendments or comparable ethical standards.

\section{Data Collection}

Demographic information was gathered for all study participants. Additional data collected for patients with PD included disease course, Hoehn and Yahr stage (H\&Y), UPDRS scores, and daily Levodopa Equivalent Dose (LED). Dyskinesia and motor fluctuations (MF) were recorded based on part four of the UPDRS. Moreover, the Non-Motor Symptom Questionnaire (NMSQ) was used to screen for salivation, 
hyposmia, constipation, and frequent urination. The Hamilton Depression Scale (HAMD) and Hamilton Anxiety Scale (HAMA) scaled the depression and anxiety. The Epworth Sleepiness Scale (ESS), PSQI, and Parkinson's Disease Quality of Life Scale-8 (PDQ-8) were used to evaluate subjective sleepiness, sleep quality, and quality of life, respectively. Fatigue, autonomic dysfunction, ability to perform activities of daily living, and cognitive function were assessed using the FSS, Scales for Outcomes in Parkinson's Disease-Autonomic Dysfunction (SCOPA-AUT), Ability of daily living scale (ADLs), and the Mini Mental State Examination (MMSE), respectively.

\section{Diagnosis of sarcopenia}

Patients exhibiting decreased muscle strength or physical function combined with decreased muscle mass were diagnosed with sarcopenia, in accordance with the Asian Working Group for Sarcopenia 2019 Consensus on the Diagnosis and Treatment of Sarcopenia[21].

\section{Muscle strength}

Grip strength was measured using a Camry Hand Dynamometer (Guangdong Xiangshan Weighing Apparatus, Zhongshan City, Guangdong Province). Measurements were obtained with the patient in the standing position and with the elbow in extension. Maximum isometric strength was measured for the dominant hand or both hands, and the largest reading after two tests was selected. Low muscle strength was defined as $<28.0 \mathrm{~kg}$ in men and $<18.0 \mathrm{~kg}$ in women.

\section{Physical function}

Physical function was assessed via a 6-meter gait speed (GS) test. The average speed during the time required to walk $6 \mathrm{~m}$ at a normal gait speed was recorded from the beginning of movement, without considering acceleration or deceleration. Two tests were performed, taken the average value for analysis. A pace of $\leq 6 \mathrm{~s}$ was considered to reflect decreased physical function.

\section{Muscle mass}

Multi-frequency bioelectrical impedance analysis (BIA) was performed to determine each patient's appendicular skeletal mass (ASM). All patients underwent assessment after fasting and excretion in the morning. Low muscle mass was defined as $<7.0 \mathrm{~kg} / \mathrm{m}^{2}$ in men and $<5.7 \mathrm{~kg} / \mathrm{m}^{2}$ in women.

\section{Statistical analysis}

All statistical analyses were showed by the Statistical Package for the Social Sciences version 24.0 (SPSS Co., Chicago, IL, USA). Measurement data conforming to the normal distribution are showed as the mean \pm standard deviation (SD), while those with a non-normal distribution as the median (interquartile range). Categorical parameters were expressed using frequencies and percentages. Differences in continuous variables among study groups came to assess using Student's t-test or the Mann-Whitney Utest, while differences in the distribution of categorical variables taking use of the chi-square test. Multiple logistic regression analysis was performed to examine the relationship between NMS and sarcopenia. Receiver operating characteristic (ROC) analysis was performed in order to investigate the 
diagnostic value about sleep quality and fatigue severity in the identification of sarcopenia. A $p$ value of $<0.05$ was treated statistically significant.

\section{Results}

\section{Clinical and demographic data}

A total of 140 patients with PD and 38 healthy controls were enrolled in our study, and 123 patients were included in the final analysis (Fig. 1). All participants were of Han Chinese descent. The PD group included 56 men and 67 women. Average ages in the PD and control groups were $65.73 \pm 9.36$ years and $67.03 \pm 10.89$ years, respectively. Sarcopenia was observed in $33(26.8 \%)$ patients with PD and four (10.4\%) control participants, and the incidence of sarcopenia was significantly higher in the PD group than in the control group $\left(x^{2}=4.357, p=0.035\right)$. Body mass index (BMI) $(p=0.009)$ and grip strength $(p<0.001)$ were lower in patients with PD than in controls, but the two groups exhibited non-significant differences in ASM $(p=0.075)$. Gait speed was also slower in the PD group than in the control group $(p=0.021)$. The comparison of demographic characteristics and sarcopenia parameters is presented in Table 1. 
Table 1

Demographic characteristics and sarcopenia parameters of patients with idiopathic PD and control group.

\begin{tabular}{|c|c|c|c|c|}
\hline Variable & Control(n=38) & $P D(n=123)$ & $t / x^{2} / z$ & $P$ Value \\
\hline Age, y & $67.03 \pm 10.89$ & $65.73 \pm 9.36$ & 0.716 & 0.475 \\
\hline Sarcopenia, n(\%) & $4(10.5)$ & $33(26.8)$ & 4.359 & 0.037 \\
\hline Education ,y & $6.5(5,9)$ & $7(4,9)$ & -0.292 & 0.770 \\
\hline Male,n(\%) & $23(60.5)$ & $56(45.5)$ & 2.613 & 0.106 \\
\hline Hypertension, n (\%) & $9(23.7)$ & $34(27.7)$ & 0.232 & 0.630 \\
\hline Type 2 diabetes, n (\%) & $5(13.2)$ & 13(10.6) & 0.196 & 0.658 \\
\hline Smoker, n (\%) & $6(15.8)$ & $24(19.5)$ & 0.265 & 0.606 \\
\hline Alcohol user, n (\%) & $7(18.4)$ & 21(17.1) & 0.037 & 0.848 \\
\hline Tea or coffee user,n (\%) & $10(26.3)$ & $33(26.8)$ & 0.004 & 0.950 \\
\hline $\mathrm{BMI}\left(\mathrm{kg} / \mathrm{m}^{2}\right)$ & $25.19 \pm 2.58$ & $23.64 \pm 3.32$ & 2.638 & 0.009 \\
\hline Skeletal muscle mass index & $7.24(6.57,7.86)$ & $6.83(5.91,7.70)$ & -1.178 & 0.075 \\
\hline Grip strength & $25.35(20.65,33.6)$ & $22.60(18.05,30.55)$ & -2.307 & 0.021 \\
\hline $6 \mathrm{~m}$ GS & $6.02(5.36,6.83)$ & $7.26(6.41,8.48)$ & -4.459 & $凶 0.001$ \\
\hline \multicolumn{5}{|c|}{ Values in bold are significant at $P<0.05$} \\
\hline
\end{tabular}

\section{Clinical features of PD with and without sarcopenia}

A total of 33 participants in the PD group had sarcopenia, while the remaining 90 did not. The demographic and clinical characteristics of patients with PD with and without sarcopenia are summarised in Table 2. The sarcopenia group included 11 men and 22 women, although the difference in gender was not significant $(p=0.1)$. Sarcopenia was more common in patients with advanced PD $(p=0.019)$ but was not correlated with disease duration, motor symptoms, or LED. Our analysis also indicated that patients with PD who had sarcopenia were older and had poorer nutritional status and ADL function, lower BMI, higher UPDRS scores (parts I, II, and IV), and poorer quality of life than those with PD who did not have sarcopenia. In addition, we observed significantly higher NMS burden in the sarcopenia group. A multiple logistic regression analysis adjusted for age, sex, nutritional status, HY stage, and BMI revealed that the occurrence of sarcopenia was significantly associated with the FSS score $(B=1.097$, wald $=4.134,95 \% \mathrm{Cl}: 1.003$ to $1.199, p=0.042$ ). Sarcopenia also exhibited a significant positive correlation with the PSQI score $(B=1.305$, wald $=4.694,95 \% \mathrm{Cl}: 1.026$ to $1.662, p=0.03)$ (Table 3). 
Table 2

Comparison of basic clinical data, motor and non-motor symptoms of PD patients with or without sarcopenia

\begin{tabular}{|c|c|c|c|c|}
\hline \multirow[t]{2}{*}{ Variable } & \multicolumn{2}{|c|}{ Without Sarcopenia With Sarcopenia } & \multirow[t]{2}{*}{$t / x^{2} / z$} & \multirow[t]{2}{*}{ P Vaule } \\
\hline & $(n=90)$ & $(n=33)$ & & \\
\hline Age,y & $63.37 \pm 8.99$ & $72.18 \pm 7.12$ & -4.209 & $凶 0.001$ \\
\hline Disease duration,y & $4(2,7)$ & $6(3,9.5)$ & -1.772 & 0.076 \\
\hline Education background,y & $7.5(4.75,9)$ & $6(2,9.5)$ & -0.460 & 0.646 \\
\hline Male,n(\%) & $45(50)$ & 11(33.3) & 2.705 & 0.100 \\
\hline Hypertension, n (\%) & $27(30)$ & $7(21.2)$ & 0.932 & 0.334 \\
\hline Type 2 diabetes, n (\%) & $11(12.2)$ & $2(6.1)$ & 0.970 & 0.325 \\
\hline Smoker, n (\%) & $20(22.2)$ & $4(12.1)$ & 1.569 & 0.210 \\
\hline Alcohol user, n (\%) & 17(18.9) & $4(12.1)$ & 0.781 & 0.377 \\
\hline Tea or coffee user,n (\%) & 25(27.8) & $8(24.2)$ & 0.154 & 0.695 \\
\hline Types of Motor Symptoms & & & 0.795 & 0.672 \\
\hline Tremor-dominant, n (\%) & $28(31.1)$ & $8(24.2)$ & & \\
\hline PIGD-dominant, n (\%) & $50(55.6)$ & 19(57.6) & & \\
\hline Mixed, n (\%) & 12(13.3) & $6(18.2)$ & & \\
\hline nutrition & & & 23.819 & 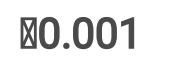 \\
\hline Good nutrition, n (\%) & $52(57.8)$ & $8(24.2)$ & & \\
\hline Risk of malnutrition, n (\%) & $37(41.1)$ & 17(51.6) & & \\
\hline Malnutrition, n (\%) & $1(1.1)$ & $8(24.2)$ & & \\
\hline $\mathrm{BMI}\left(\mathrm{kg} / \mathrm{m}^{2}\right)$ & $24.74 \pm 2.72$ & $20.64 \pm 2.96$ & 7.225 & 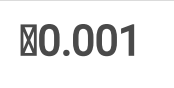 \\
\hline Skeletal muscle mass index & 7.36(6.38,7.89) & $5.5(5.18,6.66)$ & -6.336 & $\mathbb{\nabla 0 . 0 0 1}$ \\
\hline Grip strength & $24.9(18.7,32.63)$ & $18.7(15.3,22.05)$ & -4.167 & $\mathbb{\otimes 0 . 0 0 1}$ \\
\hline $6 \mathrm{~m} \mathrm{GS}$ & $7.04(5.99,8.33)$ & 7.75(7.15,9.43) & -2.934 & 0.003 \\
\hline UPDRS-I & $3(2,5)$ & $4(2.5,6)$ & -2.056 & 0.040 \\
\hline
\end{tabular}

Values in bold are significant at $P<0.05$

Data were expressed as $n(\%)$, mean $\pm S D$, median (interquartile range). $P$ values are from Student' $s$ ttest or the Mann-Whitney U-test or Chi-square tests 


\begin{tabular}{|c|c|c|c|c|}
\hline \multirow[t]{2}{*}{ Variable } & \multicolumn{2}{|c|}{ Without Sarcopenia With Sarcopenia } & \multirow[t]{2}{*}{$t / x^{2} / z$} & \multirow[t]{2}{*}{ P Vaule } \\
\hline & $(n=90)$ & $(n=33)$ & & \\
\hline UPDRS-II & $11(8,16.25)$ & $15(9,22.5)$ & -2.501 & 0.012 \\
\hline UPDRS-III & $24(15,33)$ & $26(13.5,33)$ & -0.231 & 0.817 \\
\hline UPDRS-IV & $1(0,3)$ & $2(0.5,6)$ & -2.793 & 0.005 \\
\hline Dyskinesia, n (\%) & 11(12.2) & $6(18.2)$ & 0.72 & 0.396 \\
\hline Motion fluctuations, n (\%) & $20(22.2)$ & 13(39.4) & 3.627 & 0.057 \\
\hline H-Y stage, n (\%) & & & 10.954 & 0.052 \\
\hline 1.0 & 15(16.7) & $5(15.2)$ & & \\
\hline 1.5 & $11(12.2)$ & $2(6.1)$ & & \\
\hline 2.0 & $20(22.2)$ & $10(30.3)$ & & \\
\hline 2.5 & $25(27.8)$ & $3(9)$ & & \\
\hline 3.0 & $16(12.2)$ & $8(24.2)$ & & \\
\hline 4.0 & $3(3.3)$ & $5(15.2)$ & & \\
\hline $\mathrm{H}-\mathrm{Y}$ early stage, $\mathrm{n}(\%)$ & $71(78.9)$ & $20(60.6)$ & 4.193 & 0.041 \\
\hline LEDD & $387.5(225,654.75)$ & $499(293.75,724)$ & -1.369 & 0.171 \\
\hline MMSE & $26(23,28)$ & $23(19.5,27)$ & -2.446 & 0.014 \\
\hline PSQI & $8(5.75,11)$ & $13(10,15.5)$ & -4.693 & $\llbracket 0.001$ \\
\hline ESS & $4(3,9)$ & $7(3,12.5)$ & -1.891 & 0.059 \\
\hline FSS & $39.5(27.75,48.25)$ & $49(43.5,53.5)$ & -3.815 & $凶 0.001$ \\
\hline HAMA & $8(5,13)$ & $16(10.5,21)$ & -4.328 & $凶 0.001$ \\
\hline HAMD & $9(6,13.25)$ & $17(12.5,22.5)$ & -4.157 & $凶 0.001$ \\
\hline NMSQ & $8(5,12.25)$ & $12(8,17)$ & -3.383 & 0.001 \\
\hline SCOPA-AUT & $10(6,14)$ & $15(10.5,19.5)$ & -3.297 & 0.001 \\
\hline PDQ-8 & $8.5 \pm 4.81$ & $12.39 \pm 5.57$ & -3.81 & 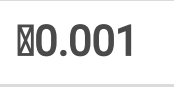 \\
\hline ADL & $24(21,35)$ & $37(25.5,56)$ & -3.753 & $凶 0.001$ \\
\hline \multicolumn{5}{|c|}{ Values in bold are significant at $P<0.05$} \\
\hline $\begin{array}{l}\text { Data were expressed as } n \\
\text { test or the Mann-Whitney } U\end{array}$ & $\begin{array}{l}\text { an } \pm S D \text {, median (interc } \\
\text { Chi-square tests }\end{array}$ & tile range). $P$ valu & e from & dent's t- \\
\hline
\end{tabular}


Table 3

Multivariate logistic regression analysis of sarcopenia in PD patients

\begin{tabular}{|lll|}
\hline multivariate analysis & Model 1 & Model 2 \\
\cline { 2 - 3 } & OR(95\% Cl) & OR(95\% Cl) \\
\hline MMSE & $0.950(0.873,1.034)$ & $1.022(0.881,1.885)$ \\
\hline FSS & $1.059(1.009,1.111)$ & $1.097(1.003,1.119)$ \\
\hline HAMA & $1.184(1.030,1.361)$ & $1.305(1.026,1.662)$ \\
\hline HAMD & $0.999(0.904,1.105)$ & $0.974(0.84,1.13)$ \\
\hline NMSQ & $1.003(0.909,1.106)$ & $1.004(0.873,1.155)$ \\
\hline SCOPA & $0.975(0.870,1.092)$ & $0.860(0.636,1.162)$ \\
\hline BMI & $1.051(0.957,1.156)$ & $1.169(0.957,1.427)$ \\
\hline Age & & $0.388(0.232,0.646)$ \\
\hline Nutrition & & $1.183(1.058,1.322)$ \\
\hline Gender & & $0.252(0.047,1.348)$ \\
\hline HY stage & & $1.426(0.292,6.964)$ \\
\hline $\begin{array}{l}\text { Model 1: Multiple logistic regression analysis unadjusted; Model 2: Multiple logistic regression } \\
\text { analysis adjusted for gender, age (in years, SD corrected), Nutrition, and HY stage, SD corrected for } \\
\text { continuous variables. }\end{array}$ & $0.831(0.156,4.431)$ \\
\hline
\end{tabular}

\section{Optimal cut-off value for discriminating sarcopenia in PD}

ROC curve analysis was used to evaluate the potential ability of NMS to distinguish sarcopenia in PD. The area under the curve (AUC) values were 0.725 (95\% Cl: 0.637 to $0.802 ; p<0.001)$ for the FSS score and 0.776 (95\% Cl: 0.692 to $0.846 ; p<0.001)$ for the PSQI score. Further analyses of diagnostic performance revealed that the FSS could differentiate sarcopenia in PD with a sensitivity of $87 \%$ and a specificity of $50 \%$, while the sensitivity and specificity values for the PSQI were $72.7 \%$ and $74.4 \%$ respectively (Figure 2). However, joint use of FSS and PSQI increased the predictive value (AUC $=0.804$, $p<0.001$ ) (Figure 3).

\section{Discussion}

To date, few studies have investigated sarcopenia in patients with PD, especially within the Han population. This is the first study to report that sarcopenia is strongly and independently associated with symptoms of fatigue and severe disturbances in sleep quality in Han Chinese patients with PD. Our results also indicated that the incidence of sarcopenia was greatly higher in patients with PD than in the 
healthy controls, which is accordance with with the results reported in previous studies[15]. Furthermore, our ROC analysis indicated that combined use of FSS and PSQI scores may be a valuable screening method for identifying sarcopenia in patients with PD.

Our analysis confirmed that the appearance of sarcopenia is significantly associated with the age of the patient, which is consistent with the definition of sarcopenia as a geriatric disease and published geriatric literature[22, 23]. Previous studies have demonstrated that older adults often experience a reduction in skeletal muscle mass, which is considered to be related to nervous system alterations and changes in metabolism, hormone levels, nutrition, and physical activity[23, 24]. These changes in muscle mass and function can lead to disability, reduced mobility, functional dependence, and functional decline[25-27]. Similarly, in our study, patients with PD who had sarcopenia exhibited poorer nutritional status and lower BMI than their counterparts without sarcopenia. From a clinical perspective, these results highlight the need to screen for sarcopenia in older adults with PD and address changes in nutritional status and BMI as early as possible in the treatment process.

Notably, although many patients with PD in our study were in the early stage of the disease, a loss of muscle mass was still observed in these patients. Furthermore, in accordance with the findings of Tamer et al.[28], we observed significant decreases in muscle mass with disease progression. These decreases in muscle mass exert a negative impact on patient prognosis, emphasizing the importance of early detection and intervention.

The incidence of sarcopenia was also higher in our sample of patients with PD than in the general older adult population in China[29]. This may be related to the existence of common pathophysiological pathways affected by PD and sarcopenia, including those associated with inflammation, autophagy, oxidative stress, and apoptosis[17, 30, 31]. Several studies have indicated that high levels of interleukin-6 (IL-6), are related to the loss of muscle mass and lower body function, as well as the incidence of older adults[32, 33]. Some studies have also reported that IL-6 levels and signalling pathways are imbalanced in patients with neurodegenerative diseases[30], and higher serum IL-6 levels have been associated with a slower gait in patients with PD. In addition, these patients exhibit extreme difficulty in maintaining balance. Therefore, increases in IL- 6 levels may lead to weakness and fatigue in patients with PD, further contributing to functional decline.

Changes in brain structure and brain networks are thought to exert a great influence on the pathophysiology of sarcopenia in patients with PD. In their study of patients with PD, Wu et al. observed that decreased mass in core thigh muscles was associated with lower grey matter volumes in the left superior temporal gyrus and right hook gyrus, longer disease duration, and female sex. The reduction of network capacity in the default mode leads to insufficient activity of the task-related network during task execution, which leads to low motor function[34].

In addition, Drey et al.[17] reported that the UPDRS-III score is significantly related to early sarcopenia, suggesting that there may be a common pathway in the preclinical stage of the two diseases, and that sarcopenia may be a sign of PD in the preclinical stage. Caviness[35] further observed that, when 
compared with healthy controls, patients with PD exhibited significant decreases in the number of motor units in the hand muscles. Another study by Drey et al.[36] indicated that the number of motor neurons is reduced in older adults with sarcopenia, suggesting that neurodegeneration plays a key role in the pathogenesis of sarcopenia.

Sarcopenia may also be affected by hormonal changes in patients with PD. Androgens play an important role in maintaining muscle mass, and low plasma testosterone levels may lead to the development or progression of sarcopenia[37]. However, no studies have explored the relationship between testosterone levels and sarcopenia in patients with PD, necessitating further research for clarification. In addition, gastrointestinal infections and the use of levodopa may impact muscle mass in patients with PD[38]. Further, the motor dysfunction associated with PD may lead to significant decreases in muscle mass and physical ability relative to levels observed in healthy older adults[28].

Our findings indicated that sarcopenia as determined based on BIA findings is negatively associated with sleep quality and the severity of fatigue in patients with PD. Sleep and fatigue are the two main NMS of PD. Further, the prevalence of sleep disorders in patients with PD is $47.7-89.1 \%$, which is higher than that in the general population[39]. Sleep disorders in patients with PD may be due to degeneration of thalamocortical pathways and alterations in neurotransmitter levels[40]. While the exact mechanism underlying the link between sarcopenia and sleep disorders remains unclear, several potential explanations have been proposed. Recent research has indicated that sleep might exert an important influence on muscle protein metabolism[41]. During aging, individuals experience decreases in the duration and quality of sleep as well as the circadian rhythm is disturbed, and the prevalence of sleep disorders is increasing. This situation favours proteolysis and may lead to changes in body composition that addition the hazard of insulin resistance. In addition, studies have suggested that levels of growth hormone (GH), insulin-like growth factor-1 (IGF-1), and testosterone play a role in the association between sarcopenia and sleep disorders[42]. Decreased physical activity levels in patients with PD may represent another mechanism underlying the link between sarcopenia and sleep disorders. In this study, we did not evaluate the relationship between physical activity and endocrine hormone levels, and next research is required to explore whether mechanisms associated with these variables can explain the link between sleep disorders and sarcopenia in patients with PD. Treating sleep disorders, normalising circadian rhythms and sleep homeostasis, and maintaining good sleep quality may provide a tactics for maintaining or restoring muscle health in these patients.

Fatigue refers to the subjective experience of a lack of energy and physical exhaustion, which is common in patients with $\mathrm{PD}[43]$ and can bring about the quality of life go down. Studies have also reported a close relevance between muscle mass and fatigue in patients with cancer[44], suggesting that decreases muscle mass may be a critical factor in the development of fatigue. We speculate that early evaluation and management of skeletal sarcopenia may better the quality of life of patients with PD by reducing symptoms of fatigue. Given that sleep and fatigue may be involved in the association between PD and sarcopenia, we attempted to determine whether FSS and PSQI scores had enough discriminative power to distinguish sarcopenia in patients with PD. ROC curve analysis revealed that the AUC values for the FSS 
and PSQI were 0.725 and 0.776 , respectively, while that for combined use of the FSS and PSQI reached 0.804. These results indicate that FSS and PSQI scores exhibit acceptable sensitivity and specificity for the potential discrimination of sarcopenia in patients with PD.

The present study had some limitations. First, the study was executed at the PD Clinic in Suzhou, where eating habits and ethnicity are relatively homogenous. Therefore, our findings may not be applicable to other, more diverse populations, highlighting the need for multicentre studies to confirm our results. Second, by excluding patients who were unable to cooperate with the assessments and those in the late stages of PD who could not stand on their own, we may have underestimated the prevalence of sarcopenia, as these excluded patients may have had more severe features than included patients. In addition, we did not use objective tools for assessments of sleep. In future studies, polysomnography (PSG) can be used to better evaluate and analyse the relationship between sleep characteristics and sarcopenia in patients with PD. Finally, the cross-sectional nature of our study allows us to report only the association of sarcopenia with patient fatigue and sleep, but we cannot state the direction of this relationship. Long-term follow-up studies and cohort studies are required further explore these relationships.

\section{Conclusion}

The present findings demonstrate that the prevalence of sarcopenia is greatly higher in patients with PD than in the general population in China. Moreover, in the PD group, patients with sarcopenia had worse sleep quality and more severe fatigue than those without. Therefore, interventions for addressing decreases in muscle mass in the early stages of PD are urgently required.

\section{Abbreviations}

PD

Parkinson's Disease

NMS

non-motor symptoms

ROC

receiver operating characteristic

OR

odds ratio

$\mathrm{Cl}$

confidence interval

PSQI

Pittsburgh Sleep Quality Index

AUC

area under the curve

FSS 


\section{Fatigue Severity Scale}

UPDRS

Unified Parkinson's Disease Rating Scale

$\mathrm{H} \& \mathrm{Y}$

Hoehn and Yahr stage

LED

Levodopa Equivalent Dose

MF

motor fluctuations

NMSQ

Non-Motor Symptom Questionnaire

HAMD

Hamilton Depression Scale

HAMA

Hamilton Anxiety Scale

ESS

Epworth Sleepiness Scale

PDQ-8

Parkinson's Disease Quality of Life Scale-8

SCOPA-AUT

Scales for Outcomes in Parkinson's Disease-Autonomic Dysfunction

ADLs

Ability of daily living scale

MMSE

Mini Mental State Examination

GS

gait speed

BIA

bioelectrical impedance analysis

ASM

appendicular skeletal mass

SD

standard deviation

BMI

Body mass index

IL-6

interleukin-6

$\mathrm{GH}$

growth hormone

IGF-1 
insulin-like growth factor-1

PSG

polysomnography.

\section{Declarations}

\section{Ethics approval and consent to participate}

All participants should provide written informed consent in person, and the study won the approval of the Ethics Review Committee of The Second Affiliated Hospital of Soochow University (approval number: LK-2018-061-01). All methods were carried out in accordance with relevant guidelines and regulations.

\section{Consent for publication}

Not applicable.

\section{Availability of data and materials}

The datasets used and analysed during the current study are available from the corresponding author on reasonable request.

\section{Competing interests}

The authors declare no conflicts of interest.

\section{Funding}

This work was supported by the Jiangsu Provincial Key R\&D Program (BE2018658), Suzhou Clinical Research Center of Neurological Disease (Szzx201503), Discipline Construction Program of the Second Affiliated Hospital Soochow University (XKTJ-XK202001, XKTJ-RC202004) and the Priority Academic Program Development of Jiangsu Higher Education Institutions (PAPD). Gusu Health Talents Training Project(GSWS2019041, GSWS2020035). Corresponding author Prof. Chun-feng Liu is a grant recipient for these program.

\section{Authors' contributions}

CFL, CJM, and QWL designed the study, QWL, ZHL, RFS, YCZ, PZ collected the data, QWL analyzed the data and drafted the manuscript, CJM revised the article, CFL guided and supervised the whole process. All authors have read and approved the manuscript.

\section{Acknowledgements}

None.

\section{Authors' information}


${ }^{1}$ Department of Neurology and Clinical Research Center of Neurological Disease, the Second Affiliated Hospital of Soochow University, Suzhou 215004, China

${ }^{2}$ Department of Neurology, Affiliated Hefei Hospital of Anhui Medical University, Hefei 230011, China

${ }^{3}$ Institute of Neuroscience, Soochow University. Suzhou 215004, China

\section{References}

1. Dorsey ER, Elbaz A, Nichols E, Abd-Allah F, Abdelalim A, Adsuar JC, et al. Global, regional, and national burden of Parkinson's disease, 1990-2016: a systematic analysis for the Global Burden of Disease Study 2016. Lancet Neurol. 2018;17:939-53.

2. Reeve A, Simcox E, Turnbull D. Ageing and Parkinson's disease: Why is advancing age the biggest risk factor? Ageing Res Rev. 2014;14:19-30.

3. Martinez-Martin P. The importance of non-motor disturbances to quality of life in Parkinson's disease. J Neurol Sci. 2011;310:12-6.

4. Martinez-Martin P. Nonmotor symptoms and health-related quality of life in early Parkinson's disease: NONMOTOR SYMPTOMS AND HRQOL IN PD. Mov Disord. 2014;29:166-8.

5. Angulo J, El Assar M, Rodríguez-Mañas L. Frailty and sarcopenia as the basis for the phenotypic manifestation of chronic diseases in older adults. Mol Aspects Med. 2016;50:1-32.

6. Musso CG, Jauregui JR, Macías Núñez JF. Frailty phenotype and chronic kidney disease: a review of the literature. Int Urol Nephrol. 2015;47:1801-7.

7. Montano-Loza AJ. Clinical relevance of sarcopenia in patients with cirrhosis. WJG. 2014;20:8061.

8. Meza-Junco J, Montano-Loza AJ, Baracos VE, Prado CMM, Bain VG, Beaumont C, et al. Sarcopenia as a Prognostic Index of Nutritional Status in Concurrent Cirrhosis and Hepatocellular Carcinoma. J Clin Gastroenterol. 2013;47:861-70.

9. Peball M, Mahlknecht P, Werkmann M, Marini K, Murr F, Herzmann $\mathrm{H}$, et al. Prevalence and Associated Factors of Sarcopenia and Frailty in Parkinson's Disease: A Cross-Sectional Study. Gerontology. 2019;65:216-28.

10. Rosenberg IH. Stalking Sarcopenia. Ann Intern Med. 1995;123:727.

11. Lim S, Kwon S-Y, Yoon JW, Kim SY, Choi SH, Park YJ, et al. Association Between Body Composition and Pulmonary Function in Elderly People: The Korean Longitudinal Study on Health and Aging. Obesity. 2011;19:631-8.

12. Sarodnik C, Bours SPG, Schaper NC, van den Bergh JP, van Geel TACM. The risks of sarcopenia, falls and fractures in patients with type 2 diabetes mellitus. Maturitas. 2018;109:70-7.

13. Tang T-C, Hwang A-C, Liu L-K, Lee W-J, Chen L-Y, Wu Y-H, et al. FNIH-defined Sarcopenia Predicts Adverse Outcomes Among Community-Dwelling Older People in Taiwan: Results From I-Lan Longitudinal Aging Study. The Journals of Gerontology: Series A. 2018;73:828-34. 
14. Vetrano DL, Pisciotta MS, Laudisio A, Lo Monaco MR, Onder G, Brandi V, et al. Sarcopenia in Parkinson Disease: Comparison of Different Criteria and Association With Disease Severity. J Am Med Dir Assoc. 2018;19:523-7.

15. Peball M, Mahlknecht P, Werkmann M, Marini K, Murr F, Herzmann H, et al. Prevalence and Associated Factors of Sarcopenia and Frailty in Parkinson's Disease: A Cross-Sectional Study. Gerontology. 2019;65:216-28.

16. Lima DP, de Almeida SB, Bonfadini J, de C, de Luna, de Alencar JRG, Pinheiro-Neto MS. EB, et al. Clinical correlates of sarcopenia and falls in Parkinson's disease. PLoS ONE. 2020;15:e0227238.

17. Drey M, Hasmann SE, Krenovsky J-P, Hobert MA, Straub S, Elshehabi M, et al. Associations between Early Markers of Parkinson's Disease and Sarcopenia. Front Aging Neurosci. 2017;9.

18. Lee C-Y, Chen H-L, Chen P-C, Chen Y-S, Chiang P-L, Wang C-K, et al. Correlation between Executive Network Integrity and Sarcopenia in Patients with Parkinson's Disease. IJERPH. 2019;16:4884.

19. Cai Y, Feng F, Wei Q, Jiang Z, Ou R, Shang H. Sarcopenia in Patients With Parkinson's Disease: A Systematic Review and Meta-Analysis. Front Neurol. 2021;12:598035.

20. Tan AH, Hew YC, Lim S-Y, Ramli NM, Kamaruzzaman SB, Tan MP, et al. Altered body composition, sarcopenia, frailty, and their clinico-biological correlates, in Parkinson's disease. Parkinsonism Relat Disord. 2018;56:58-64.

21. Chen L-K, Woo J, Assantachai P, Auyeung T-W, Chou M-Y, lijima K, et al. Asian Working Group for Sarcopenia: 2019 Consensus Update on Sarcopenia Diagnosis and Treatment. Journal of the American Medical Directors Association. 2020;21:300-307.e2.

22. Cruz-Jentoft AJ, Landi F, Topinková E, Michel J-P. Understanding sarcopenia as a geriatric syndrome: Current Opinion in Clinical Nutrition and Metabolic Care. 2010;13:1-7.

23. Doherty TJ. Invited Review: Aging and sarcopenia. J Appl Physiol. 2003;95:1717-27.

24. Welle S. Cellular and Molecular Basis of Age-Related Sarcopenia. Can J Appl Physiol. 2002;27:1941.

25. Janssen I, Heymsfield SB, Ross R. Low Relative Skeletal Muscle Mass (Sarcopenia) in Older Persons Is Associated with Functional Impairment and Physical Disability. J Am Geriatr Soc. 2002;50:88996.

26. Lauretani F, Russo CR, Bandinelli S, Bartali B, Cavazzini C, Di lorio A, et al. Age-associated changes in skeletal muscles and their effect on mobility: an operational diagnosis of sarcopenia. J Appl Physiol. 2003;95:1851-60.

27. Landi F, Liperoti R, Russo A, Giovannini S, Tosato M, Capoluongo E, et al. Sarcopenia as a risk factor for falls in elderly individuals: Results from the ilSIRENTE study. Clin Nutr. 2012;31:652-8.

28. Yazar T, Yazar HO, Zayimoğlu E, Çankaya S. Incidence of sarcopenia and dynapenia according to stage in patients with idiopathic Parkinson's disease. Neurol Sci. 2018;39:1415-21.

29. Huang C-Y, Hwang A-C, Liu L-K, Lee W-J, Chen L-Y, Peng L-N, et al. Association of Dynapenia, Sarcopenia, and Cognitive Impairment Among Community-Dwelling Older Taiwanese. Rejuven Res. 
2016;19:71-8.

30. Scalzo P, Kümmer A, Cardoso F, Teixeira AL. Serum levels of interleukin-6 are elevated in patients with Parkinson's disease and correlate with physical performance. Neurosci Lett. 2010;468:56-8.

31. Kwan P. Sarcopenia, a Neurogenic Syndrome? J Aging Res. 2013;2013:1-10.

32. Pereira LSM, Narciso FMS, Oliveira DMG, Coelho FM, Souza DG de, Dias RC. Correlation between manual muscle strength and interleukin-6 (IL-6) plasma levels in elderly community-dwelling women. Arch Gerontol Geriatr. 2009;48:313-6.

33. Cesari M, Penninx BWJH, Pahor M, Lauretani F, Corsi AM, Williams GR, et al. Inflammatory Markers and Physical Performance in Older Persons: The InCHIANTI Study. The Journals of Gerontology Series A: Biological Sciences and Medical Sciences. 2004;59:M242-8.

34. Wu Y-N, Chen M-H, Chiang P-L, Lu C-H, Chen H-L, Yu C-C, et al. Associations between Brain Structural Damage and Core Muscle Loss in Patients with Parkinson's Disease. JCM. 2020;9:239.

35. Caviness JN, Smith BE, Clarke Stevens J, Adler CH, Caselli RJ, Hentz JG, et al. Motor unit number estimates in idiopathic Parkinson's disease. Parkinsonism Relat Disord. 2002;8:161-4.

36. Drey M, Krieger B, Sieber CC, Bauer JM, Hettwer S, Bertsch T, et al. Motoneuron Loss Is Associated With Sarcopenia. J Am Med Dir Assoc. 2014;15:435-9.

37. Basualto-Alarc $\tilde{A}^{3} n$ Varela C, Duran D, Maass J, Estrada R. M. Sarcopenia and Androgens: A Link between Pathology and Treatment. Front Endocrinol. 2014;5.

38. Adams F, Boschmann M, Lobsien E, Kupsch A, Lipp A, Franke G, et al. Influences of levodopa on adipose tissue and skeletal muscle metabolism in patients with idiopathic Parkinson's disease. Eur $\mathrm{J}$ Clin Pharmacol. 2008;64:863-70.

39. Liu C-F, Wang T, Zhan S-Q, Geng D-Q, Wang J, Liu J, et al. Management Recommendations on Sleep Disturbance of Patients with Parkinson's Disease. Chin Med J. 2018;131:2976-85.

40. Falup-Pecurariu C, Diaconu Ş. Sleep Dysfunction in Parkinson's Disease. In: International Review of Neurobiology. Elsevier; 2017. pp. 719-42.

41. Piovezan RD, Abucham J, dos Santos RVT, Mello MT, Tufik S, Poyares D. The impact of sleep on agerelated sarcopenia: Possible connections and clinical implications. Ageing Res Rev. 2015;23:210-20.

42. Buchmann N, Spira D, Norman K, Demuth I, Eckardt R, Steinhagen-Thiessen E, Sleep, Muscle Mass and Muscle Function in Older People: A Cross-Sectional Analysis Based on Data From the Berlin Aging Study II (BASE-II). Deutsches Aerzteblatt Online. 2016. https://doi.org/10.3238/arztebl.2016.0253.

43. Grace J, Mendelsohn A, Friedman JH. A comparison of fatigue measures in Parkinson's disease. Parkinsonism Relat Disord. 2007;13:443-5.

44. Wang B, Thapa S, Zhou T, Liu H, Li L, Peng G, et al. Cancer-related fatigue and biochemical parameters among cancer patients with different stages of sarcopenia. Support Care Cancer. 2020;28:581-8. 
Figures

\section{Figure 1}

Flow diagram of the study participants.

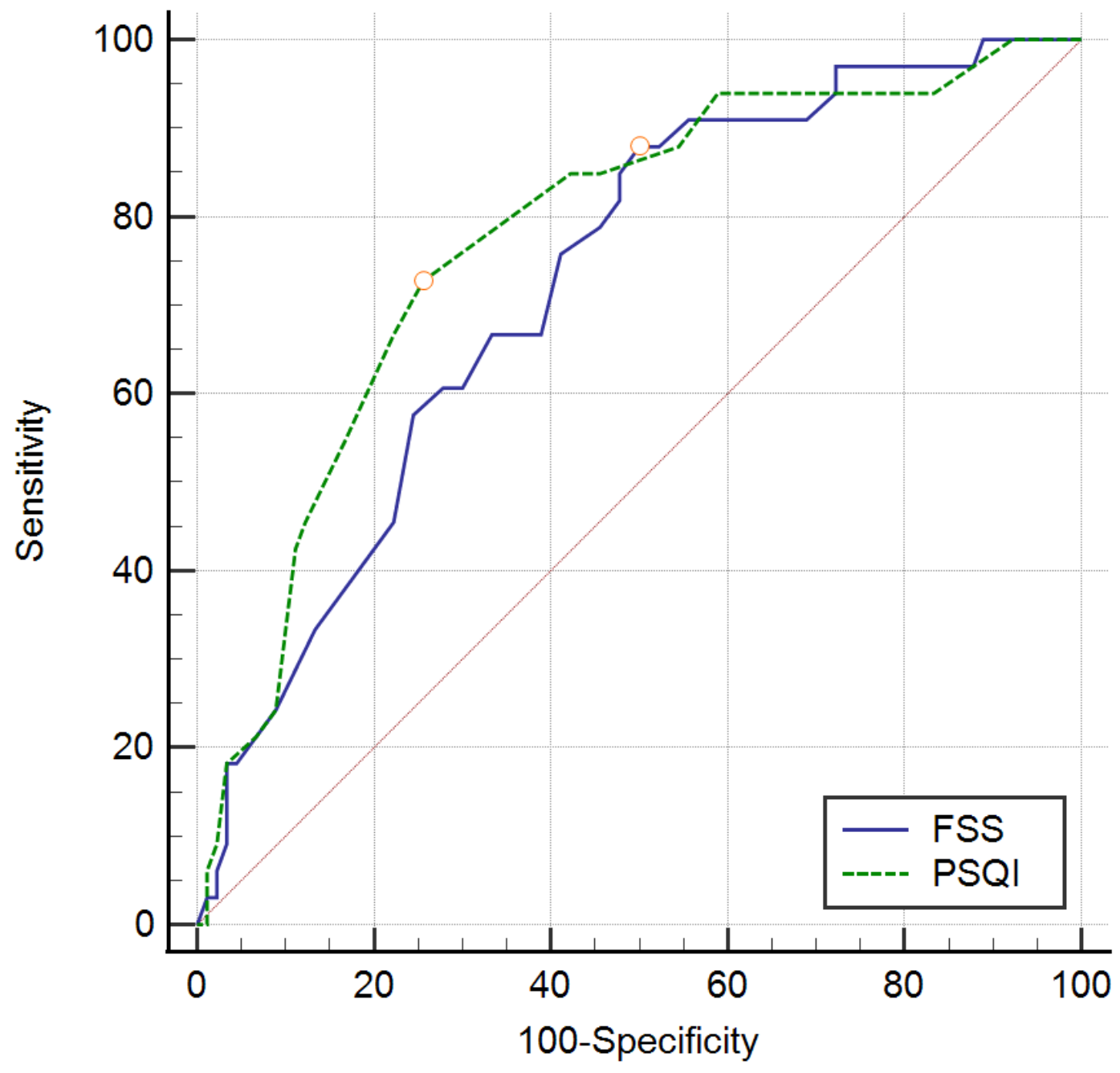

Figure 2

ROC showed the best cut-off value of fatigue severity score and PSQI score to distinguish sarcopenia in $\mathrm{PD}$ 


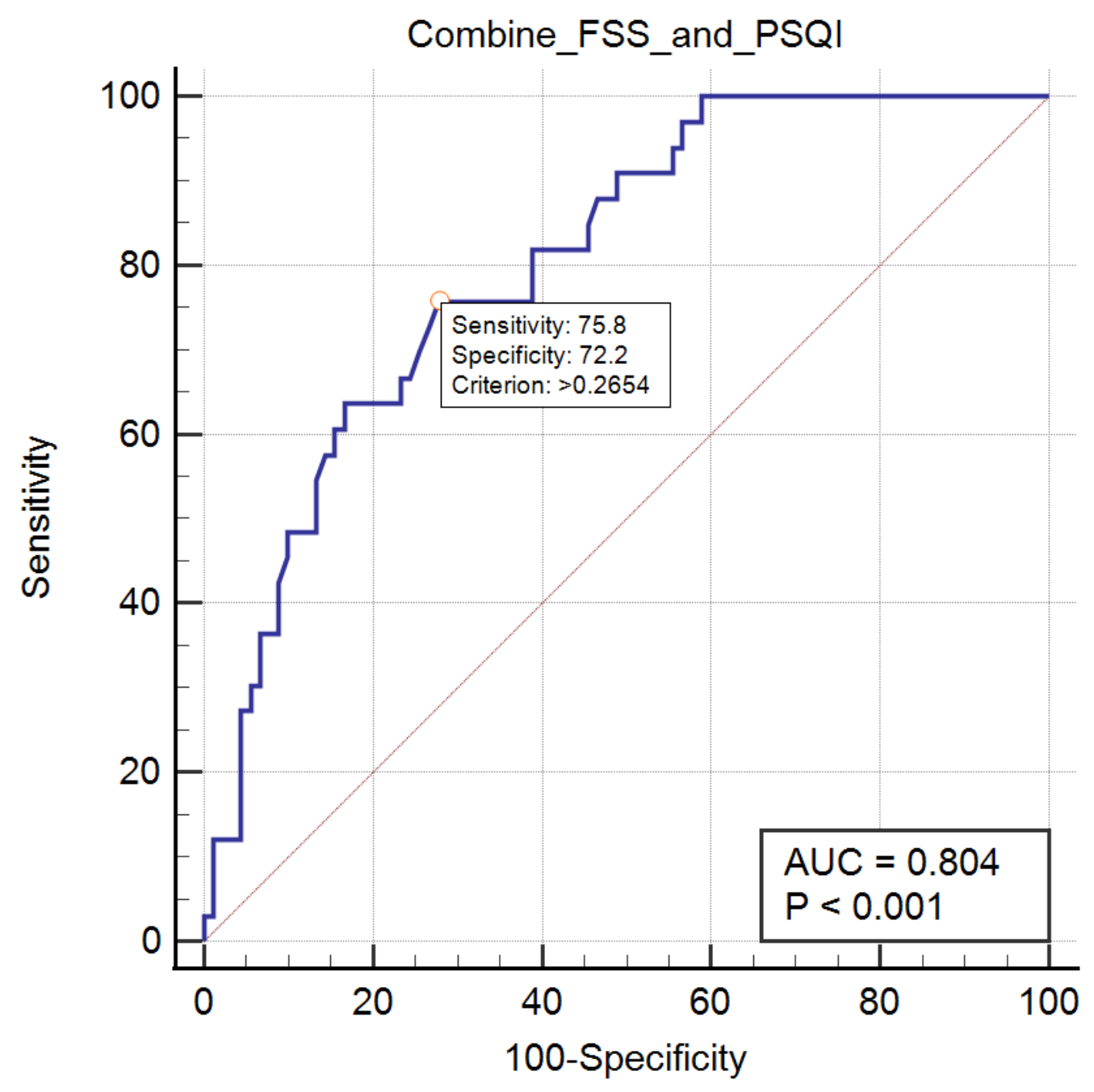

Figure 3

The ROC of the joint use of FSS and PSQI analysis showed an AUC value of 0.804 